\title{
Educación artística y competencia mediática en el currículo de Educación Primaria
}

\author{
Antonia Ramírez GARCÍA \\ Universidad de Córdoba \\ ed1ragaa@uco.es \\ Paula RenÉs Arellano \\ Universidad de Cantabria \\ paula.renes@unican.es \\ Jacqueline SÁNCHEZ CARRERO \\ Universidad de Huelva \\ jsanchezcarrero@gmail.com
}

\begin{abstract}
Resumen
En este trabajo se realiza un análisis de la presencia de la competencia mediática en los objetivos, contenidos y criterios de evaluación del área de Educación Artística en la etapa de Educación Primaria. La metodología utilizada auna una perspectiva cualitativa y cuantitativa. Los resultados indican que la competencia mediática se evidencia en todos los elementos curriculares que integran esta área. Finalmente, se ofrecen propuestas un mejor desarrollo integral del alumnado vinculado a los medios de comunicación.
\end{abstract}

Palabras clave: Competencia Mediática; Educación Artística; Educación Primaria.

\section{Arts Education and media competence in the curriculum of primary education}

\begin{abstract}
This paper is an analysis of the presence of media competence in the objectives, content and evaluation criteria of Arts Education subject in Primary Education. The methodology combines qualitative and quantitative perspective. The results indicate that media competence is evident in all curricular elements that compose this subject. Finally, suggestions are offered better overall development of students linked to the media.
\end{abstract}

Keywords: Media Competence; Art Education; Elementary Education.

\section{Referencia normalizada:}

Ramírez García, A.; Renés Arellano, P.; Sánchez Carrero, J. (2013) Educación artística y competencia mediática en el currículo de Educación Primaria. Historia y Comunicación Social. Vol. 18 No Especial Octubre. Págs. 673-686.

Sumario: 1. El cuándo, cómo, dónde... de la educación mediática. 1.1. Análisis de la competencia mediática en el currículo escolar. 1.2. La educación artística en el currículo escolar. 2. Metodología. 3. Resultados. 4. Conclusiones. 5. Referencias bibliográficas. 6. Notas. 


\section{El cuándo, cómo, dónde ... de la Educación Mediática}

Educación y comunicación audiovisual parecen tener cada vez más sentido en la sociedad de la información en la que vivimos. En los últimos años recientes investigaciones y experiencias han fomentado la incorporación de la competencia mediática en las aulas escolares. Pero no ha sido hasta el año 2008 cuando el Parlamento Europeo realizó una aproximación etimológica del concepto educación mediática y su significado, reseñando la importancia de su desarrollo en las edades tempranas dentro de las instituciones sociales, principalmente la escuela y la familia.

En España, se iniciaron las primeras investigaciones de la mano de Ferrés (2007) a través de un acuerdo el Consell de l' Audiovisual de Catalunya (CAC) y la Universidad Pompeu Fabra. La finalidad fue medir y establecer dimensiones de análisis de la competencia mediática en nuestro país (Ferrés, 2006).

Posteriormente, surgió un nuevo trabajo de investigación en el que se describieron las diferentes investigaciones llevadas a cabo por 17 universidades españolas en materia de competencia mediática y en la que se detectaron la necesidad de una formación en medios entre los ciudadanos que les permitiera progresar como personas críticas y responsable ante las pantallas (Ferrés et al., 2011).

Tanto los niños como los adolescentes viven inmersos entre pantallas, muchos de ellos son los denominados nativos digitales. Sin embargo, tener acceso a recursos audiovisuales o aparatos electrónicos no significa ser competentes ante ellos. Este tipo de colectivo carece de criterios que les pueda ayudar ser consumidores responsables y críticos con los medios que utilizan.

El nuevo reto es lograr que los ciudadanos sean capaces de desarrollar capacidades y estrategias que les permitan no solamente consumir medios, sino producirlos, crearlos, diseñarlos, ser agentes activos de los procesos de construcción de significados digitalizados, fomentando así prosumidores inteligentes.

Asimismo, el proceso que se ha seguido para formular esos programas de formación de ciudadanos prosumidores, parte de la primera reunión acerca de la alfabetización mediática centrada en la Declaración de Grünwald (1982); del Informe Delors el cual hacía referencia a los pilares de la educación: aprender a conocer, a hacer, a convivir y a ser; y, finalmente, la Agenda de París (2007) en la cual se hace pública la necesidad de desarrollar iniciativas de educación mediática entre el profesorado y el alumnado de todos los niveles educativos.

En relación con un estudio realizado en Inglaterra para evaluar la educación mediática entre el colectivo de educación primaria (Strudley, 2008), mostró que el alumnado posee capacidades y puede desarrollar estrategias suficientes para disponer de los medios de comunicación, pero al mismo tiempo se convierten en usuarios pasivos de los medios. Un avance de ese estudio reveló que el alumnado era capaz de crear sus propios materiales multimedia lo que indica ese grado de iniciativa que se debería profundizar desde el ámbito formal educativo. 


\subsection{Análisis de la competencia mediática en el currículo escolar}

Con la Ley Orgánica 2/2006, de 3 de mayo, de Educación (LOE), se incorporan al currículo de la etapa obligatoria ocho competencias básicas: competencia matemática; competencia en comunicación lingüística; competencia en el conocimiento y la interacción con el mundo físico; competencia social y ciudadana; competencia cultural y artística; tratamiento de la información y competencia digital; competencia para aprender a aprender; autonomía e iniciativa personal.

Aquella competencia vinculada a los objetivos de este trabajo se sustenta, en un primer momento, en la competencia denominada del "tratamiento de la información y la competencia digital", entendida como:

El tratamiento de la información y la competencia digital implica ser una persona autónoma, eficaz, responsable, crítica y reflexiva al seleccionar, tratar y utilizar la información y sus fuentes, así como las distintas herramientas tecnológicas; también tener una actitud crítica y reflexiva en la valoración de la información disponible, contrastándola cuando es necesario, y respetar las normas de conducta acordadas socialmente para regular el uso de la información y sus fuentes en los distintos soportes (LOE, 2006).

Posteriormente, el Real Decreto 1513/06, de 7 de diciembre, desarrolla esta competencia básicas y todo el currículo de la etapa de educación primaria.

Sin embargo, el concepto de competencia mediática aborda muchos más elementos que los meramente digitales, también engloba aspectos de análisis sobre comprensión, escritura, creación, innovación, diseño o conocimiento del entorno, que no quedan recogidos en la competencia sobre el tratamiento de la información y la competencia digital. Por tal motivo, consideramos necesario realizar un análisis pormenorizado de cómo están presentes los elementos de la competencia mediática en cada una de las áreas curriculares de educación primaria. Se intenta así favorecer la construcción de un desarrollo curricular integral, en el que la competencia mediática esté adecuadamente integrada y en la que se eduque en, con y antes los medios (Bernabéu, 2011). Este trabajo se presenta como contribución a dicha construcción, realizando un análisis de la presencia de factores que componen la educomunicación en el currículo del área de educación artística.

\subsection{La educación artística en el currículo}

La educación artística está integrada en el currículo educación primaria y es definida como "un conjunto de saberes cuyo objetivo es la creación de hábitos orientados a la producción y a la interpretación de los productos y fenómenos" (Moreno, 2005: 14). Estos saberes según el Real Decreto 1513/06, de 7 de diciembre, engloban dos lenguajes, por un lado el plástico y por el otro el musical.

Tanto la percepción como la expresión, son lenguajes propios de la educación artística y ayudan al niño a desarrollar sus capacidades sobre el reconocimiento visual, 
corporal, auditivo y sensorial del entorno, a manifestar sus ideas y hacer un uso de las diferentes técnicas artísticas. Asimismo, estos lenguajes, expresión y percepción, han sido dos conceptos empleados en el diseño de los criterios de medición de la competencia mediática en nuestro país (Ferrés y Piscitelli, 2012).

La realidad actual es que el trabajo en el aula puede seguir modelos tradicionales o por el contrario, transformadores del conocimiento (Vahter, 2012) que favorecen el desarrollo integral de la formación de los docentes (De Backer, Lombaerts, Peeters y Elias, 2012). Muestra de ello es el e-Teatrix for kids que permite al alumnado expresar sus sentimientos y experiencias sobre cualquier aspecto educativo, incluyendo métodos empleados por los docentes (Pan, Cheok, Yang, Zhu y Shi, 2006). Otro ejemplo es el Maps of Time a través del cual se utilizan técnicas artísticas antiguas en un mundo virtual y la difusión de producciones a través de los medios de comunicación (Rusu, 2012).

Algunos autores como Botella et al. (2013) o Colbert y Courchesne (2012), consideran que los medios audiovisuales son fundamentales en el proceso creativo de las personas y contribuyen a la creación de nuevos productos (Coskun, 2010).

\section{Metodología}

La investigación realizada presenta una naturaleza cualitativa e intenta responder a dos grandes cuestiones: ¿está presente la competencia mediática en el currículo de educación primaria?, ¿cómo contribuye el área de educación artística al desarrollo de la competencia mediática? y ¿qué relación puede establecerse con las seis dimensiones establecidas por Ferrés y Piscitelli (2012) para la mencionada competencia?

Asimismo, a través de esta investigación damos respuesta a una de las tareas del Proyecto I+D La enseñanza obligatoria ante la competencia en comunicación audiovisual en un entorno digital (EDU2010-21395-C03-03) acerca de la evaluación de la competencia mediática en el entorno escolar.

El procedimiento seguido ha sido el siguiente:

1. Localización de la normativa que regula el currículo en la etapa de educación primaria en las diferentes comunidades autónomas.

2. Tratamiento de la información hallada a través del programa ATLAS.ti.

3. Análisis de los objetivos, contenidos y criterios de evaluación del currículo del segundo ciclo de la etapa de educación primaria en el área de educación artística en todas las comunidades autónomas. La elección del ciclo se debió a que en cuarto curso se celebran las pruebas de evaluación diagnóstica.

4. Establecimiento de relaciones entre los elementos curriculares y las dimensiones de la competencia mediática establecidas por Ferrés y Piscitelli (2012): Lenguajes, Tecnología, Procesos de interacción, Procesos de producción y 
difusión, Ideología y valores, y Estética, teniendo en consideración los dos ámbitos que componen cada una de ellas - el análisis de contenido y la expresión-.

En la última fase del procedimiento se ha requerido la colaboración de diez maestros y maestras en ejercicio ${ }^{1}$. El criterio establecido para contemplar la relación entre los elementos curriculares y las dimensiones de la competencia era la concordancia entre los participantes -miembros del proyecto responsables de esta tarea y profesorado colaborador- en, almenos, un $70 \%$ de los casos.

\section{Resultados}

Aquellas investigaciones y estudios orientados a la relación de currículo y competencia mediática coinciden en que existe una escasez de las mismas y una inexistencia de la competencia mediática en el currículo educativo (Tucho, 2008; Camps, 2009; Aparici, Campuzano, Ferrés y García, 2010).

La competencia mediática se ha definido en torno a 55 descriptores distribuidos en seis dimensiones. En lo relacionado con el área de educación artística, la cuantificación de la relación existente entre dicha competencia y los elementos curriculares se establece en la tabla 1.

Tabla 1. Presencia de la competencia mediática en los elementos curriculares del área de Educación Artística.

\begin{tabular}{l|l|c|c|c|} 
Dimensiones competencia mediática \\
Descriptores
\end{tabular}

Fuente: Elaboración propia.

Como se puede observar, la presencia de la competencia mediática en los objetivos del área de educación artística es de un 18,18\%, llegando a un 30,91\% en los contenidos del segundo ciclo y destacando el descenso al $12,73 \%$ en los criterios de evaluación. En la tabla 1 se puede apreciar que todas las dimensiones de la competencia quedan presentes en alguno de los elementos curriculares del área de educación artística en el segundo ciclo de la etapa de educación primaria. Se puede destacar que la dimensión procesos de producción y difusión se halla presente únicamente 
en los contenidos. Asimismo, las dimensiones más representadas en los elementos curriculares son lenguajes y estética. Sin embargo, se ha de señalar que esta última dimensión, como se indicará más adelante, apenas está presente en las comunidades autónomas.

De forma más detallada, la distribución de los porcentajes entre las dimensiones y subdimensiones de la competencia mediática se muestra en la figura 1. A través de ella se muestra las relaciones existentes entre los elementos curriculares -objetivos, contenidos y criterios de evaluación- y las dimensiones, subdimensiones y descriptores de la competencia mediática.

En cuanto a los objetivos del área de educación artística que se destacan son:

1. Observar, analizar e interpretar imágenes fijas y móviles del entorno en los distintos medios de comunicación para favorecer la comprensión crítica de los significados y valores que transmiten sus mensajes y para conocer algunas de las posibilidades de los medios audiovisuales y las nuevas tecnologías, y utilizarlos como recursos para la elaboración de producciones propias, ya sea de forma autónoma o en combinación con otros medios y materiales (L2a/I1e).

Las comunidades que lo asumen reelaborándolo son Cantabria y Cataluña, y Valencia añadiéndole "descubrir significados de interés expresivo y estético".

2. Identificar, comprender y utilizar los elementos del lenguaje visual y algunos conceptos de sintaxis que configuran los códigos de las creaciones plásticas y de las imágenes presentes en nuestro entorno (L1a).

Este objetivo es fijado por Cantabria y reelaborado en Cataluña.

3. Conocer algunas de las posibilidades de los medios audiovisuales y de las tecnologías de la información y la comunicación en los que intervienen la imagen y el sonido, y utilizarlos como recursos para la observación, la búsqueda de información y la elaboración de producciones propias, ya sea de forma autónoma o en combinación con otros medios. (T1a / V1a).

La normativa de Andalucía, Aragón, Asturias, Cantabria, Castilla y León, Cataluña, Extremadura, Galicia, La Rioja, Madrid, Navarra y Valencia contemplan este objetivo.

4. Indagar en las posibilidades del sonido, la imagen y el movimiento como elementos de representación y comunicación y utilizarlas para expresar, valorar y aceptar hechos, ideas y sentimientos, contribuyendo con ello al equilibrio afectivo y a la ampliación de las capacidades relacionales y comunicativas (L1e).

Las siguientes comunidades autónomas lo incorporan en su currículo: Andalucía, Aragón, Asturias, Cantabria, Cataluña, Castilla La Mancha, Castilla y León, Galicia, Islas Baleares y Navarra. La comunidad de Aragón insiste en la ampliación de las capacidades de relación y comunicación a partir de dicho objetivo. Por su parte, Asturias hace hincapié en la relación con los demás en condiciones de igualdad, Cataluña lo reelabora con otras palabras y Galicia lo completa con otro objetivo más. 
Figura 1. Distribución de la presencia de los elementos curriculares del área de Educación Artística en las dimensiones y subdimensiones de la competencia mediática.

\begin{tabular}{|c|c|c|c|c|c|}
\hline \multicolumn{2}{|c|}{$\begin{array}{l}\text { Dimensiones y } \\
\text { subdimensiones }\end{array}$} & Descriptores & $\mathbf{O}$ & $\mathbf{C}$ & $\mathrm{CE}$ \\
\hline \multirow{5}{*}{ 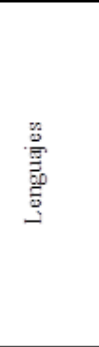 } & \multirow{3}{*}{ 㖪 } & $\begin{array}{l}\text { L1a. Capacidad de interpretar y de valorar los diversos códigos de } \\
\text { representación y la función que cumplen en un mensaje. }\end{array}$ & $\mathrm{x}$ & $\mathrm{x}$ & $\mathrm{x}$ \\
\hline & & $\begin{array}{l}\text { L2a. Capacidad de analizar y de valorar los mensajes desde la } \\
\text { perspectiva del significado y del sentido, de las estructuras narrativas y } \\
\text { de las convenciones de género y de formato. }\end{array}$ & $x$ & $\mathrm{x}$ & \\
\hline & & $\begin{array}{l}\text { L3a. Capacidad de establecer relaciones entre tex tos, códigos y medios, } \\
\text { elaborando conocimientos abiertos, sistem atizados e interrelacionados. }\end{array}$ & & $\mathrm{x}$ & \\
\hline & \multirow{2}{*}{ 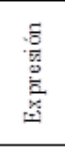 } & $\begin{array}{l}\text { L le. Capacidad de expresarse mediante una amplia gama de sistem as de } \\
\text { representacióny de significación. }\end{array}$ & $\mathrm{x}$ & & \\
\hline & & $\begin{array}{l}\text { L2e. Capacidad de elegir entre distintos sistem as de representación y } \\
\text { distintos estilos en función de la situación comunicativa, del tipo de } \\
\text { contenido que hay que transmitiry del tipo de interlocutor. }\end{array}$ & & & $x$ \\
\hline \multirow{4}{*}{$\frac{\frac{\pi}{80}}{\frac{0}{9}}$} & \multirow{2}{*}{ 笣 } & $\begin{array}{l}\text { T1a Comprensión del papel que desempeñan en la sociedad las } \\
\text { tecnologias de la información y de la comunicación y de sus posibles } \\
\text { efectos. }\end{array}$ & $x$ & $\mathrm{x}$ & \\
\hline & & $\begin{array}{l}\text { T2 a Capacidad de manejo de las innovaciones tecnológicas que hacen } \\
\text { posible una comunicación multimodal y multim edial. }\end{array}$ & & $x$ & \\
\hline & \multirow{2}{*}{ 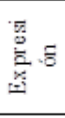 } & $\begin{array}{l}\text { T1 e. Capacidad de manejar con corrección herramientas comunicativas } \\
\text { en un entorno multimedial y multimodal. }\end{array}$ & $\mathrm{x}$ & & $\mathrm{x}$ \\
\hline & & $\begin{array}{l}\text { T2e. Capacidad de elaborar y de manipular imágenes y sonidos desde la } \\
\text { conciencia de cómo se construyen las representaciones de la realidad. }\end{array}$ & & $\mathrm{x}$ & \\
\hline \multirow{3}{*}{ 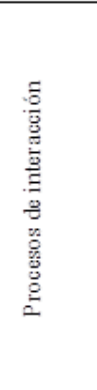 } & \multirow{2}{*}{ 㖪 } & $\begin{array}{l}\text { I1 a. Capacidad de dilucidar por qué gustan unos medios, unos productos } \\
\text { o unos contenidos, por qué tienen éxito, individual o colectivamente: } \\
\text { qué necesidades } \\
\text { y deseos satisfacen en } 10 \text { sensorial, en } 10 \text { emotivo, en lo cognitivo, en } 10 \\
\text { estético, en lo cultural, etc. }\end{array}$ & & $\mathrm{x}$ & \\
\hline & & $\begin{array}{l}\text { I2a. Capacidad de valorar los efectos cognitivos de las emociones: } \\
\text { tomar conciencia de las ideas y valores que se asocian con personajes, } \\
\text { acciones y situaciones que generan, según los casos, emociones } \\
\text { positivas y ne gativas. }\end{array}$ & & $\mathrm{x}$ & \\
\hline & 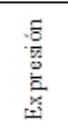 & $\begin{array}{l}\text { I1 e. Actitud activa en la interacción con las pantallas, entendidas como } \\
\text { oportunidad para construir una ciudadania más plena, un desarrollo } \\
\text { integral, para transformarse y para transformar el entorno. }\end{array}$ & $\mathrm{x}$ & & \\
\hline 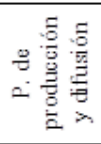 & 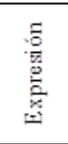 & $\begin{array}{l}\text { Ple. Capacidad de compartir y diseminar información, a través de los } \\
\text { medios tradicionales y de las redes sociales, incrementando la } \\
\text { visibilidad de los mensajes, en interacción con comunidades cada vez } \\
\text { más amplias. }\end{array}$ & & $x$ & \\
\hline \multirow{2}{*}{ 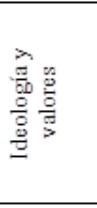 } & \multirow{2}{*}{ 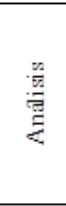 } & $\begin{array}{l}\text { V1a. Habilidad para buscar, organizar, contrastar, priorizar y sintetizar } \\
\text { informaciones procedentes de distintos sistemas y de diferentes } \\
\text { entornos. }\end{array}$ & $x$ & $\mathrm{x}$ & $x$ \\
\hline & & $\begin{array}{l}\text { V2a. Capacidad de detectar las intenciones o intereses que subyacen } \\
\text { tanto en las producciones corporativas como en las populares, asi como } \\
\text { su ideologia y valores, explicitos o latentes, adoptando una actitud } \\
\text { critica ante ellos. }\end{array}$ & $\mathrm{x}$ & $\mathrm{x}$ & \\
\hline \multirow{4}{*}{ 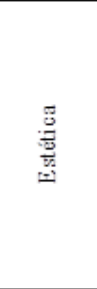 } & \multirow{2}{*}{ 器 } & $\begin{array}{l}\text { E1 a Capacidad de relacionar las producciones mediáticas con otras } \\
\text { manife staciones artisticas, detectando influencias mutuas. }\end{array}$ & $\mathrm{x}$ & $\mathrm{x}$ & $\mathrm{x}$ \\
\hline & & $\begin{array}{l}\text { E2 a Sensibilidad para reconocer una producción mediática que no se } \\
\text { adecue a unas exigencias mirimas de calidad estética. }\end{array}$ & & & $x$ \\
\hline & \multirow{2}{*}{ 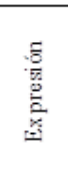 } & $\begin{array}{l}\text { E1e. Capacidad de producir mensajes elementales que sean } \\
\text { comprensibles y que contribuyan a incrementar los niveles personales o } \\
\text { colectivos de creatividad, originalidad y sensibilidad. }\end{array}$ & $\mathrm{x}$ & $\mathrm{x}$ & \\
\hline & & $\begin{array}{l}\text { E2e. Capacidad de apropiarse y de tranformar producciones artisticas, } \\
\text { potenciando la creatividad, la innovación, la experimentación y la } \\
\text { sensibilidad estética. }\end{array}$ & & $\mathrm{x}$ & $\mathrm{x}$ \\
\hline
\end{tabular}

$\mathrm{O}=$ Objetivos; $\mathrm{C}=$ Conteridos; $\mathrm{CE}=$ Criterios de evaluación.

Fuente: Elaboración propia. 
5. Desarrollar confianza en las elaboraciones artísticas propias, disfrutando con su realización y apreciando su contribución al crecimiento personal y del grupo, para afianzar su autoestima y mejorar la capacidad de expresión de ideas y sentimientos. (E1e)

Este objetivo lo propone el País Vasco y la comunidad de Asturias lo dividen en dos objetivos.

6. Aplicar los conocimientos artísticos en la observación, el análisis y la interpretación de situaciones, objetos, signos, imágenes y sonidos de la realidad cotidiana y de diferentes manifestaciones del mundo del arte y la cultura, especialmente las contemporáneas, para comprenderlas mejor y formar un gusto propio, así como para propiciar una actitud receptiva y una comprensión crítica del fenómeno artístico (V2a).

La comunidad de Cantabria lo hace suyo y Galicia lo reelabora insistiendo en la actitud crítica y selectiva ante la información que se recibe.

En relación con los contenidos del área de educación artística, los ejemplos más destacados son los siguientes:

1. Análisis y valoración de la intención comunicativa de los nuevos códigos audiovisuales, de las imágenes en los medios y TIC (L3a).

Este contenido está presente en las comunidades de Cantabria, Castilla León, Cataluña y Galicia.

2. Lectura, interpretación, análisis y valoración de la información que proporcionan las imágenes en el contexto social y comunicación de las apreciaciones obtenidas: imágenes (fijas y móviles) de distintos medios -fotografía, cartel, valla publicitaria, cine, vídeo, televisión, videojuego, ordenador y TIC (L1a).

Las comunidades autónomas que lo asumen son: Andalucía, Aragón, Asturias, Canarias, Cantabria, Islas Baleares, La Rioja, Madrid, Navarra, Región de Murcia, Valencia.

3. Apreciación de algunas características (formales, materiales y tecnológicas) en la creación de objetos y de imágenes que han cambiado o se mantienen a lo largo del tiempo (T1a).

Este contenido es propuesto por la comunidad de Cataluña.

4. Descripción de imágenes (fijas y en movimiento) presentes en contextos próximos: cómics, ilustraciones, fotografias, carteles, dibujos animados, propaganda, cine, marcas,... (L2a).

Las comunidades que lo incorporan son: Andalucía, Aragón, Asturias, Canarias, Cantabria, Castilla La Mancha, Cataluña, Galicia, Islas Baleares y Navarra.

5. Observación y valoración del uso de la música en medios de comunicación y/o audiovisuales y digitales: televisión, publicidad, cine, videojuegos y programas informáticos (I1a). 
Este contenido se establece en las comunidades de Canarias, Castilla León y Cataluña.

6. Utilización de medios audiovisuales, recursos digitales, programas y recursos informáticos como registro y complemento expresivo para la creación de piezas musicales y para la sonorización de imágenes y de representaciones dramáticas, producciones artísticas,... (T2e).

Las comunidades que lo hacen propio son: Aragón, Asturias, Canarias, Cantabria, Castilla La Mancha, Cataluña, Extremadura, La Rioja, Madrid, Navarra, País vasco, Región de Murcia y Valencia.

7. Observación, análisis y valoración de imágenes y de su intención comunicativa que aparecen en los medios de comunicación y TIC (V2a).

Este contenido se aprecia en las comunidades de Aragón, que insiste en el desarrollo del espíritu crítico, Asturias, Canarias, Cantabria, Castilla León, Cataluña, Galicia, Islas Baleares, Navarra y País Vasco.

8. Empleo de las TIC para el tratamiento de imágenes, diseño y animación y para la difusión de los trabajos elaborados (P1e). Las comunidades autónomas que lo incorporan a su currículo son: Aragón, Asturias, Canarias, Cantabria, Castilla León, Galicia, Islas Baleares, La Rioja, Madrid, Navarra y Murcia.

9. Búsqueda guiada de información, imágenes y datos sobre obras de arte y autores, producciones artísticas, musicales, instrumentos, intérpretes, conciertos, estilos musicales, festivales,... empleando las TIC (V1a).

Las comunidades en las que se recoge normativamente son: Aragón, Asturias, Canarias, Cantabria, Castilla León, Cataluña, Galicia, Islas Baleares, Navarra y País Vasco.

10. Elaboración de composiciones plásticas utilizando fotografías, revistas, prensa, cromos,... (E2e).

Las comunidades que lo contemplan en su currículo son Canarias, Castilla León y Cataluña.

11. Interés por identificar la motivación en la creación de objetos y de imágenes, las distintas visiones del arte y la relación de los objetos y las imágenes con los contextos de producción artística (E1e).

Este contenido es legislado por Cataluña.

Finalmente, en lo relativo a los criterios de evaluación del área de educación artística vinculados a la competencia mediática se expresan a continuación:

1. Interpretar el contenido de imágenes y representaciones del espacio presentes en el entorno (L1a).

Las comunidades autónomas que los adoptan son Asturias y Cantabria. 
2. Desarrollar la sensibilidad estética y educacional como público al mostrar interés por conocer profesiones vinculadas a los ámbitos artísticos (E2a).

Este criterio de evaluación es propuesto por la comunidad de Canarias

3. Localizar, seleccionar y organizar información sobre manifestaciones artísticas distintas empleando las TIC (V1a). Este criterio se recoge en la normativa de Asturias, Castilla y León, Extremadura e Islas Baleares.

4. Emplear instrumentos, técnicas y materias acomodados al producto artístico (L2e).

Galicia es la comunidad que lo contempla en su normativa.

5. Realizar un proyecto artístico haciendo uso de las distintas posibilidades de transformación de materiales, texturas, formas y colores aplicados sobre diferentes soportes (E2e).

Este criterio es incorporado por la comunidad de Castilla y León a su currículo.

6. Utilizar las TIC como herramientas de expresión para crear producciones plásticas y musicales (T1e).

Las comunidades que lo incorporan al currículo son Andalucía, Asturias, Cantabria, Castilla La Mancha, Castilla y León, Cataluña, Galicia, Ibiza (resalta la valoración crítica del entorno), La Rioja y Madrid.

\section{Conclusiones}

En páginas anteriores se aludía a la escasez de investigaciones y de experiencia educativas con incorporación de la competencia mediática, bien en el ámbito curricular, bien en el desarrollo d ebueas prácticas. Pues bien, contribuyendo a la construcción de una educomunicación adecuada y con sentido, se han ofrecido unas cifras claras sobre la presencia de dicha competencia en el currículo del área de educación artística del segundo ciclo de la etapa de educación primaria. Finalmente, el análisis realizado entorno a toda la legislación autonómica ofrece conclusiones como las que se redactan a continuación:

1. Existe presencia de la competencia mediática en el currículo escolar, sin embargo, no desde criterios realistas como se desearía por los expertos en la materia. Dicha presencia se evidencia con un porcentaje significativo, ya que en todos los elementos curriculares analizados (objetivos, contenidos y criterios de evaluación) se evidencia alguna de las dimensiones establecidas por Ferrés y Piscitelli (2012).

2. En el currículo de primaria del área de educación artística, aquellos que son procesos de análisis priman sobre los expresivos en las seis dimensiones de la competencia mediática descritos. 
3. Las dimensiones lenguajes, estética y tecnología están más presentes que el resto de dimensiones en el currículo escolar.

4. La menor o mayor presencia de la competencia mediática en el currículo difiere entre las diferentes comunidades cuando se procede a su análisis discriminativo. Concretamente, Cataluña ofrece mayores posibilidades para el desarrollo de la competencia mediática en el currículo escolar que otras comunidades autónomas.

Por ende, la relevancia otorgada a la competencia mediática desde los diversos ámbitos internacionales, nacionales y autonómicas ha de contribuir al enriquecimiento del currículo escolar, ser la herramienta que permita a las personas formarse en la sociedad de la información y la comunicación. Dicha formación debe sustentarse en el desarrollo de una comunidad crítica, responsable y reflexiva ante los medios, siendo necesario, por tanto, una mayor presencia de la competencia mediática en los currículos escolares oficiales.

Siguiendo este análisis conviene replantearse las políticas educativas existentes en las que se garantice un tratamiento de la competencia mediática adecuada y equilibrada entre las distintas comunidades autónomas. Es sabido que la ausencia de un consenso en materia de educación genera sistemas incompetentes (políticos, educativos y sociales), y en este caso agrava los posibles factores de exclusión social entorno a la educomunicación.

Si bien, realizamos un llamamiento a una política equilibrada y justa, también lo hacemos por una participación activa de los ciudadanos en cuanto a no conformarse con patrones meramente receptores de información sino hacia la activación de criterios que favorezcan la actitud del prosumidor, personas creadoras y responsables de sus propias manifestaciones, al margen del tipo de lenguaje utilizado y de la comunidad autónoma en la que resida.

Asimismo, haciendo alusión al análisis de este artículo, la educación artística, por su carácter integrador e interdisciplinar, constituye un área privilegiada en el currículo escolar en la cual se pueden desarrollar las dimensiones de la competencia mediática, tal y como hemos mostrado anteriormente.

A modo de conclusión, es lógico cuestionarse ¿cómo se proyecta la presencia de la competencia mediática en el próximo currículo escolar que se está debatiendo? Aunque aún no existe una respuesta, realizando un análisis del anteproyecto de ley actual y de la presencia de la competencia mediática, se abren todavía más interrogantes en una sociedad española que continúa sin contar con un sistema educativo eficaz, de calidad y consensuado. 


\section{Referencias bibliográficas}

APARICI, R.; CAMPUZANO, A.; FERRÉS, J. y GARCÍA A. (2010): "La educación mediática en la escuela 2.0" Disponible en: http://ntic.educacion.es/w3/web 20/informes/educacion_mediatica_e20_julio20010.pdf en 23 de enero de 2013 . Consultado [21-07-2013].

BERNABÉU, N. (2011). "La educación mediática en el currículo de la loe. aportaciones de este ámbito de conocimiento a la educación por competencias básicas". Disponible en: http://es.scribd.com/doc/147584846/Bernabeu-La-educacion-mediatica-curriculo-LOE. Consultado [21-07-2013].

BOTELLA, M.; GLAVEANU, V.; ZENASNI, F.; STORME, M.; MYSZKOWSKI, N.; WOLFF, M. y LUBART, T. (2013). "How artists create: Creative process and multivariate factors", en Learning and Individual Differences, (en prensa). Disponible en: http://dx.doi.org/10.1016/j.lindif.2013.02.008. Consultado [21-07-2013].

CAMPS, V. (2009). "La educación en medios, más allá de la escuela". En: Revista Comunicar, $\mathrm{n}^{\circ}$ 32. Huelva: Grupo Comunicar, p. 139-145.

COLBERT, F. y COURCHESNE, A. (2012). "Critical issues in the marketing of cultural goods: The decisive influence of cultural transmission". En: City, Culture and Society, $\mathrm{n}^{\mathrm{o}} 3$, p. 275-280. Disponible en http://dx.doi.org/10.1016/j. ccs.2012.11.006. Consultado [03-07-2013].

COSKUN, A. E. (2010). "Experimental experience in design education as a resource for innovative thinking: The case of Bruno Munari". En Procedia-Social and Behavioral Sciences, $\mathrm{n}^{\circ}$ 2, p. 5039-5044. Disponible en doi:10.1016/j. sbspro.2010.03.817. Consultado [20-07-2013].

DE BACKER, F.; LOMBAERTS, K.; PEETERS, J. y ELIAS, W. (2012). "Visual arts as leverage for educational innovation in formal and lifelong learning". En: Procedia-Social and Behavioral Sciences, $\mathrm{n}^{\circ}$ 46, p. 1644-1648. Disponible en doi: 10.1016/j.sbspro.2012.05.354. Consultado [18-07-2013].

FERRÉS, J. (2006). La competencia en comunicación audiovisual: propuesta articulada de dimensiones e indicadores. En: Quaderns del $C A C \mathrm{n}^{\circ} 25$, Barcelona: Consell de l'Audiovisual de Catalunya, p. 9-18.

- (2007). "La competencia en comunicación audiovisual: dimensiones e indicadores". En: Revista Comunicar, no 29, Huelva: Grupo Comunicar, p. 100-107.

FERRÉS, J. y PISCITELLI, A. (2012). "La competencia en educación mediática: propuesta articulada de dimensiones e indicadores". En: Revista Comunicar, $\mathrm{n}^{\circ}$ 38, Huelva: Grupo Comunicar, p. 75-82.

FERRÉS, J. et al. (2011). Competencia mediática. Investigación sobre el grado de competencia de la ciudadanía en España. Madrid: Instituto de Tecnología Educativa, Ministerio de Educación.

LEY Orgánica 2/2006, de 3 de mayo, de Educación. Boletín Oficial del Estado, número 106, de 4 de mayo de 2006. 
MORENO, M.C. (2005). “Arte infantil en contextos educativos". En BELVER, M.H.; MORENO, C. y NUERE, S. (eds.) (2005). Arte infantil en contextos contemporáneos. Madrid: Eneida.

MUNTEAUNU, L. H. (2012). "Musical culture, a finality of musical education". En: Procedia-Social and Behavioral Sciences, $\mathrm{n}^{\circ}$ 46, 4195-4199. Disponible en doi: 10.1016/j.sbspro.2012.06.225. Consultado [12-07-2013].

PAN, Z.; CHEOK, A. D.; YANG, H.; ZHU, J. y SHI, J. (2006). "Virtual reality and mixed reality for virtual learning environments". En Computers and Graphics, $\mathrm{n}^{\circ}$ 30, p. 20-28. Disponible en: doi:10.1016/j.cag.2005.10.004. Consultado [04-072013].

REAL DECRETO 1513/2006, de 7 de diciembre, por el que se establecen las enseñanzas mínimas de Educación Primaria. Boletín Oficial del Estado $n^{\circ} 293$, de 8 de diciembre de 2006.

RUSU, A. (2012). "Revitalizing ancient technologies and advancing an ethical design in textile art education". En: Procedia-Social and Behavioral Sciences, $\mathrm{n}^{\circ}$ 51, p. 1061-1065. Disponible en: doi: 10.1016/j.sbspro.2012.08.288. Consultado [05-07-2013].

STRUDLEY, G. (2008). "The Media Literacy of Primary School Children". Disponible en: http://gdstrudley3.webs.com. Consultado [07-07-2013].

TUCHO, F. (2008). "La educación en comunicación en la LOE y sus decretos de Enseñanzas Mínimas". En Revista Comunicar, n 31, Huelva: Grupo Comunicar, p. $547-553$

VAHTER, E. (2012). "Designing the learning process in visual art classes in primary school". En: Procedia-Social and Behavioral Sciences, $\mathrm{n}^{\circ}$ 45, p. 147-157. Disponible en doi: 10.1016/j.sbspro.2012.06.551. Consultado [01-07-2013].

\section{Las autoras}

Antonia Ramírez García. Profesora contratada doctora de la Facultad de Ciencias de la Educación (Universidad de Córdoba), donde ha impartido docencia en los Grados de Maestro, licenciatura de Psicopedagogía y Másteres .Las líneas de investigación prioritarias son la evaluación formativa y de competencias, la orientación escolar y la convivencia en los centros educativos. Las últimas publicaciones se han realizado en Cultura y Educación (2013), Revista de Investigación Educativa (2012 y 2009), Revista Española de Orientación y Psicopedagogía (2012), Profesorado. Revista de currículum y formación del profesorado (2011). Recientemente ha coordinado un libro en la editorial Pirámide.

Paula Renés Arellano. Diplomada en Magisterio, Licenciada en Psicopedagogía y Profesora de la Facultad de Educación de la Universidad de Cantabria. Experta en Mediación Escolar y en TIC y Entornos Virtuales de Formación. Miembro del comité expertos en el congreso anual de Pizarra Digital que se celebra en Madrid (Uned-Pizarratic). Ha impartido formación sobre pizarra digital interactiva en El Centro de 
Formación en Nuevas Tecnologías (CeFoNT) de la Universidad de Cantabria y en el CEP de Santander. Las líneas de investigación están vinculadas con las TIC y educación mediática, así como con el análisis, gestión y resolución de conflictos y los Estilos de Aprendizaje y de Enseñanza.

Jacqueline Sánchez Carrero. Doctora en Comunicación Audiovisual por la Universidad de Sevilla, Máster en Comunicación Audiovisual en el Espacio Iberoamericano y Licenciada en Periodismo. 12 años de experiencia en TV: guión, producción y edición. Directora de "Taller Telekids", taller de educación mediática para niños. Profesora en el Máster de Comunicación y Educación Audiovisual Universidad de Huelva. Autora de los libros "Pequeños Directores: niños y adolescentes creadores de cine, vídeo y televisión" y de "Los secretos de la tele: guía de alfabetización televisiva para niños y maestros", además de numerosos artículos sobre educación mediática para niños. 